\title{
Die Zukunft der Adipositas-Chirurgie in Deutschland
}

\author{
Rudolf A. Weiner \\ Chirurgische Klinik, Krankenhaus Sachsenhausen, Frankfurt/M., Deutschland
}

Das 4. Frankfurter Meeting zur Adipositas-Chirurgie fand am 10. und 11. November 2006 wiederum im Stadtverordnetensaal des Frankfurter Römer statt. Mit einer Teilnehmerzahl von mehr als 300 Chirurgen aus allen Teilen Deutschlands zeigte sich das gewachsene Interesse an diesem sich rasch weiter entwickelnden Teilgebiet der Chirurgie. Noch nie zuvor hatten sich so viele Chirurgen aus Deutschland zusammengefunden, um sich über dieses Thema wissenschaftlich auseinanderzusetzen. Darüber hinaus waren internationale Gäste aus weiteren 10 Ländern registriert. Ehrengast der Tagung war Nicola Scopinaro, Italien, der als Nestor der internationalen Adipositas-Chirurgie die Ehrenmitgliedschaft der Gesellschaft erhielt. Neben Vorträgen aus In-und Ausland wurden wiederum Live-Operationen aus verschiedenen internationalen Operationssälen integriert.

Die Tagung wurde durch den Präsidenten der Deutschen Gesellschaft für Viszeralchirurgie (DGVS), Prof. Dr. H. Buhr (Berlin) eröffnet. Dieser sprach sich für die längst überfällige Integration der Adipositas-Chirurgie als eigenes Arbeitsgebiet in die Deutsche Gesellschaft für Viszeralchirurgie aus. Ein Antrag mit allen Unterschriften der Mitglieder der Deutschen Gesellschaft für Adipositaschirurgie wurde ihm im Rahmen der Tagung übergeben. Bislang existiert eine eigene Gesellschaft für Adipositas-Chirurgie, da es in der Vergangenheit viele Widerstände gab, diese als eigenständiges Fachgebiet unter dem Dach der Deutschen Gesellschaft für Chirurgie anzuerkennen. Die bisherigen Auffassungen, die operative Behandlung der morbiden Adipositas lediglich als Arbeitsgruppe innerhalb einer Arbeitsgemeinschaft zu integrieren, entsprechen nicht der Bedeutung dieses Arbeitsgebiets. Sie widerspricht auch allen internationalen Entwicklungen. Diese Ignoranz hat auch der Verbreitung und Weiterentwicklung der Adipositas-Chirurgie in Deutschland geschadet, das in Hinblick auf die Zahl der operativen bariatrischen Eingriffe pro Kopf der Bevölkerung noch immer einen hinteren Platz in Europa einnimmt. Immerhin ist in den USA der laparoskopische Magen-Bypass der häufigste minimal invasive Eingriff und damit häufiger als die laparoskopische Cholezystektomie.

Der Schlüssel zur Entwicklung der Adipositas in den modernen Industriegesellschaften lässt sich in den genetischen Grundlagen un- serer Stoffwechselregulation finden, der über die Jahrtausende der Menschheitsentwicklung das Überleben der Spezies gesichert hat. Während der Mensch mit dem Prinzip «Bewegung immer - Nahrung vielleicht» seine Speicherfähigkeiten für Energie ausbaute, funktioniert dieses Prinzip in der Neuzeit nicht mehr und es gilt der Leitsatz: «Nahrung immer - Bewegung vielleicht».

Die hormonalen Regulationsmechanismen weisen auch auf einen engen Zusammenhang zwischen der Entwicklung von Adipositas und Diabetes mellitus Typ II hin. Letztendlich ist die Gewichtsreduktion nicht nur die einzige Prävention, sondern auch eine effektive Behandlung für den Diabetes mellitus Typ II. Spezifische Effekte der bariatrischen Operation mit Duodenalexklusion (Bypass-Verfahren, Duodenal-Switch), hormonaler Diversion und Fundusresektion (Ghrelin-Effekt) machen eine «metabolische Chirurgie» möglich, die jeder medikamentösen Therapie oder Insulin-Applikation im therapeutischen Effekt überlegen ist. Der Diabetes mellitus Typ II kann bei Übergewicht und Adipositas durch eine operative Intervention in Form von Bypass-Verfahren schlagartig beseitigt werden, wenn noch rechtzeitig interveniert wird und ausreichend Beta-Zellen vorhanden sind. Die Insulintherapie ist beim übergewichtigen Typ-II-Diabetiker keine Therapie sui generis - im Gegenteil, sie fördert den weiteren Speicherprozess an Fett und Glykogen und führt zu weiterem Gewichtsanstieg. Mit ansteigendem Körpergewicht kommt es zu einer weiteren Steigerung des Insulinbedarfs. Ein Circulus vitiosus wird damit in Gang gesetzt, der das Leben deutlich verkürzt. Adipositas-Chirurgie ist somit auch «metabolische Chirurgie» - eine Bezeichnung, die zu einem stehenden Begriff werden sollte!

Eine Vielzahl von weiteren Stoffwechselstörungen, die unter dem Begriff «metabolischen Syndrom» zusammengefasst werden und mit der Adipositas assoziiert sind, lassen sich durch operative Maßnahmen nachhaltig beseitigen [1]. Insbesondere beim Diabetes mellitus Typ II gibt zur drastischen Gewichtsreduktion und zu den speziellen hormonalen Effekten von Bypass-Verfahren keine echten Alternativen [2].

Der direkte Zusammenhang von Adipositas und Diabetes wurde von Rosak und Weiner [3] dargestellt. Die verschiedenen operativen Verfahren sind hier in das Therapiespektrum beim Vorliegen

\section{KARGER

Prof. Dr. med. Rudolf A. Weiner 
der Kombination beider Krankheitssyndrome mit aufzunehmen. Derzeit wird an neuen interventionellen Therapieansätzen gearbeitet. Unklar bleiben allerdings die Ansätze der intermittierenden Elektrostimulation des Magens zur Beeinflussung des Diabetes mellitus Typ II. Unbeantwortet bleibt auch die Frage, ob der Gewichtsverlust oder spezifische hormonale Effekte für die therapeutisch günstige Beeinflussung des Diabetes verantwortlich sind.

Adipositas Grad III allein betrifft in Deutschland inzwischen fast $2 \%$ der Bevölkerung. Das extreme Übergewicht ist mit zahlreichen Komorbiditäten wie dem Diabetes mellitus, der Hypertonie, Herzerkrankungen, der Schlafapnoe und degenerativen Skeletterkrankungen assoziiert. Folge dieser Komorbiditäten sind weltweit jährlich mehr als 2,5 Millionen Todesfälle [4]. Das Mortalitätsrisiko steigt gegenüber dem der Normalbevölkerung um den Faktor 6-12 an, wobei die Verminderung der Lebenserwartung für übergewichtige Männer 12 Jahre und für übergewichtige Frauen 9 Jahre beträgt $[5,6]$. Die Kosten der mit der Adipositas assoziierten Begleiterkrankungen steigen ins Unermessliche, wenn alle indirekten Kosten sowie die Kosten durch die Diabetesfolgen und die medikamentöse Behandlung mitberücksichtigt werden.

Die Lebenserwartung wird besonders bei jungen adipösen Menschen durch die Adipositas deutlich verkürzt. Deshalb wird international immer nachdringlicher die frühzeitige Intervention im Kindes- und Jugendalter nicht nur diskutiert, sondern bei Extremformen immer häufiger angewandt [7]. Je frühzeitiger die Intervention, desto effektiver ist sie in Hinblick auf Vermeidung von Schäden und die Verlängerung der Lebenserwartung. Bei morbider Adipositas (Adipositas Grad III) wurde eine statistisch gesicherte Verkürzung der Lebenserwartung von 20 Jahren ermittelt [5, 6]. Christou et al. [8] konnten in einer Kohortenstudie zeigen, dass operativ behandelte morbid adipöse Patienten eine geringere Mortalitätsrate in einem Zeitraum von 5 Jahren aufwiesen als nichtoperativ behandelte $(0,68$ vs. $6,17 \%)$. Damit wurde das relative Sterblichkeitsrisiko durch die operativen Maßnahmen um $89 \%$ gesenkt (Evidenzlevel 2b). Konservative Therapieansätze beinhalten vor allem rein diätetische, pharmakologische, bewegungs- und/ oder verhaltenstherapeutische Methoden, zeigen jedoch nur kurzfristige Erfolge (Evidenzlevel 5) [9, 10]. Es existieren nur wenige Studien, die chirurgische Maßnahmen mit konservativen Therapien vergleichen. In der Studie von Andersen et al. [11] (Evidenzlevel 1) haben über einen Zeitraum von 5 Jahren 16\% der Patienten mit einer vertikalen Gastroplastik (VBG) effektiv ihr Gewicht reduzieren können, während es in der konservativ behandelten Gruppe nur 2\% waren. Noch deutlicher sind die 10-Jahres-Ergebnisse der Swedish Obesity Subject Study (SOS-Studie), die darüber hinaus den Langzeiteffekt der Gewichtsreduktion auf die Komorbiditäten belegen (Evidenzlevel 2b) [12].

Chirurgische Verfahren zur Behandlung der Adipositas Grad III zeigen in gut dokumentierten Langzeitergebnissen eine Reduktion der Komorbiditäten und eine deutlich erhöhte Lebensqualität der von der extremen Adipositas betroffenen Menschen (Evidenzlevel 2b) [5, 6, 13-15].

Die positiven Effekte der operativen Intervention bei morbider Adipositas auf Komorbiditäten, Lebensqualität und Lebenserwar- tung sind hinreichend also erwiesen. Umso mehr ist es verwunderlich, dass in Deutschland die Zahl der operativen Interventionen noch immer in einem krassen Gegensatz zur Zahl der AdipositasKranken steht. Die Zahl der operativen Eingriffe ist im Jahre 2006 leicht gestiegen, wie erste Daten aus der freiwilligen Qualitätssicherung für Adipositas-Chirurgie vermuten lassen [16, 17]. Die Ursache für die nach wie vor zu geringe Zahl von Eingriffen liegt im Genehmigungsverfahren der Krankenkassen, die sich hier von den Medizinischen Diensten beraten lassen. Allgemein weist die Finanzierung der Adipositas-Chirurgie durch Krankenkassen und Versicherungen weltweit eine große Varianz auf. In Europa nimmt Deutschland eine Sonderstellung ein, da hier die Adipositas-Chirurgie historisch eine untergeordnete Rolle spielte und die Kostenübernahme als Ausnahmeentscheidung erfolgte. Die operativen Maßnahmen zur Therapie der krankhaften Adipositas sind nicht Bestandteil des Leistungskatalogs und unterliegen somit einer Individualentscheidung, die in der Regel durch die Medizinischen Dienste der Krankenkassen getroffen. Diese orientieren sich dabei aber an internen Richtlinien, die sich nicht mit den AMWF-Richtlinien der Fachgesellschaften decken, so dass es durchaus zur Ablehnung der Kostenübernahme kommen kann, auch wenn die fachlichen Kriterien zu Operation erfüllt sind [18]. Bis zur Einführung des DRG-Systems wurde bei einer positiven Entscheidung noch die Refinanzierung nach Tagessätzen vorgenommen, weshalb die stationäre Verweildauer für Magenbandpatienten weit über dem internationalen Mittelwert lag. Die Kalkulation des DRG K04Z (Schwere Eingriffe bei morbider Adipositas) wurde auf der Basis einer kleinen Fallzahl mit dazu fehlerhaften Angaben zu Implantatkosten berechnet, sicherte jedoch auf der Grundlage der längeren Verweildauer die Durchführung der Magenbandoperationen finanziell ab. Für laparoskopische Magen-Bypass-Operationen und andere internationale Standardverfahren war damit jedoch keine finanzielle Deckung zu erreichen, weshalb eine an die Realitäten angepasste Berechnung der effektiven Kosten notwendig wurde. Für eine Trennung der einzelnen Verfahren war zunächst die exakte Klassifikation des OPS-System notwendig. Diese wurde 2005 eingeführt.

In der Häufigkeit von Adipositas-Chirurgie pro eine Million Einwohner und Jahr nimmt Deutschland einen Platz am Ende der Skala entwickelter Industrieländer ein. Haupthindernis für eine international vergleichbare Genehmigungsrate für bariatrische Eingriffe ist und bleibt die Genehmigungspraxis der Krankenkassen. Diese müssen von den vorliegenden Fakten der Effizienz chirurgischer Behandlungsmaßnahmen und damit auch von deren Kosteneffizienz Kenntnis nehmen. Eine Magen-Bypass-Operation ist bei einem insulinpflichtigen Diabetiker hinsichtlich der direkten Kosten bereits nach 8 Monaten in finanzieller Hinsicht «amortisiert». Die indirekte Kostenersparnis, wenn man die Komplikationen und Folgen des Diabetes wie diabetisches Fußsyndrom, Augen- und Nierenschädigung bis hin zu den vaskulären Komplikationen einbezieht, ist jedoch weitaus größer. Verkürzung oder Verlängerung der Lebenserwartung werden jedoch möglicherweise von manchen Versicherungsexperten anders beurteilt als von Ärzten. Die Vereinheitlichung europäischer Leitlinien ist in Angriff genommen 
worden [19]. Es bleibt zu hoffen, dass die Anpassung der Gesundheitsrechte der Bürger in Europa den auch deutschen Versicherten ein gleiches Recht auf operative Intervention bei morbider Adipositas zukommen lässt wie z. B. den Franzosen oder Belgiern.

Um für die Betroffenen in Deutschland eine international als Standard anzusehende Therapie zu ermöglichen, ist es notwendig, dass sich die Kostenträger und die Entscheidungsgremien hierzulande deutlich stärker an den medizinisch-wissenschaftlichen Leitlinien zur Therapie der Adipositas [20] orientieren. Danach ist mit einem BMI von über $35 \mathrm{~kg} / \mathrm{m}^{2}$ oder generell ab BMI von $40 \mathrm{~kg} / \mathrm{m}^{2}$ und mehr die Indikation zur operativen Intervention gegeben, wenn nicht schwere Kontraindikationen dagegensprechen. Die hohen Hürden von Vorleistungen sind jedoch entsprechend den Leitlinien zu minimieren. Adipositas-chirurgische Maßnahmen sollten bei Patienten in Betracht gezogen werden, die einen BMI $\geq 35 \mathrm{~kg} / \mathrm{m}^{2}$ mit bereits nachweisbaren Begleit- und Folgeerkrankungen oder einen $\mathrm{BMI} \geq 40 \mathrm{~kg} / \mathrm{m}^{2}$ aufweisen und bei denen konservative Behandlungsmaßnahmen nachweislich nicht erfolgreich waren. Wenngleich die Leitlinien es derzeit in Deutschland noch nicht haben festschreiben können, bleibt die Frage brennend aktuell, ob man nicht den Typ-II-Diabetikern mit einem BMI von $32 \mathrm{~kg} / \mathrm{m}^{2}$ die Chance zur unmittelbaren und sicheren Lösung zur Beseitigung seines metabolischen Syndroms und seiner Adipositas geben sollte.
Die internationalen Ergebnisse und auch nationale Studien werden hoffentlich dazu beitragen, dass es auch den von morbider Adipositas betroffenen deutschen Patienten immer mehr vergönnt sein wird, durch die effektiven Verfahren der modernen Chirurgie eine Ausweg aus ihrer Krankheitssituation zu erfahren. Etwa 1200 Eingriffe in Deutschland im Jahre 2005 bei mehr als 1000000 potentiellen Patienten mit morbider Adipositas ergibt aktuell eine jährliche Operationsfrequenz von weniger als $0,1 \%$.

Im Jahre 2006 wurden in den USA etwa 250000 bariatrische Eingriffe durchgeführt. Wenn die Häufigkeit der Eingriffe in Deutschland in den Folgejahren beibehalten wird, dann hat jedoch etwa nur 1\% aller morbid Adipösen die Chance einmal während ihres Lebens einen solchen Eingriff zu erhalten. Die Zunahme der Adipositas-Prävalenz in den nächsten Jahren bleibt damit noch unberücksichtigt. Um eine den USA adäquate Operationsfrequenz zu erreichen, wäre eine Steigerung der Operationszahlen in Deutschland um den Faktor 20-40 notwendig. Wir hoffen, dass mit dieser Tagung ein erneuter Impuls für Deutschland gegeben werden konnte. Das 5. Frankfurter Meeting findet 2008 statt. Einen besonderen Impuls sollte die deutsche Adipositas-Chirurgie durch die Vergabe des Weltkongresses der IFSO 2011 nach Hamburg erhalten.

\section{Literatur}

1 Engl J, Hanusch-Enserer U, Prager R, Patsch JR, Ebenbichler C: Das metabolische Syndrom. Auswirkungen einer ausgeprägten Gewichtsabnahme mittels chirurgischer Intervention. Wien Klin Wochenschr 2005;8:243-54.

2 Pories WJ, Swanson MS, Mc Donald KG, Long SB, Morris PG, Brown BM, Barakat HA, de Ramon RA, Israel G, Dolezal JM: Who would have thought it? An operation proves to be the most effective therapy for adult-onset diabetes mellitus. Ann Surg 1995;222:339-350.

3 Rosak C, Weiner RA: Adipositas-Chirurgie und Diabetes. Chir Gastroenterol 2007;23(suppl 1): DOI 10.1159/000098462.

4 Deitel M: Overweight and obesity worldwide now estimated to involve 1.7 billion people. Obes Surg 2003;13:329-330.

5 Fontaine KR, Barofsky I: Obesity and health-related quality of life. Obes Rev 2001;2:173-182.

6 Fontaine KR, Redden DT, Wang C, Westfall AO, Allison DB: Years of life lost due to obesity. JAMA 2003;289:187-93.

7 Inge TH, Krebs NF, Garcia VF, Skleton JA, Guice KS, Strauss RS, Albanese CT, Brandt MT, Hammer LD, Harmon CM, Kane TD, Klish JW, Oldham KT, Rudloph CD, Helmrath MA, Donovan E, Daniels SR: Bariatric surgery for severely overweight adolescents. Concerns and recommendations. Pediatrics 2004;114:217-223.
8 Christou N, Sampalis J, Liberman M, Look D, Auger S, McLean A, MacLean LD: Surgery decreases long-term mortality, morbidity and health care use in morbidly obese patients. Ann Surg 2004;240:416-24.

11 Andersen T, Pedersen BH, Dissing I, Astrup A, Henriksen JH: A randomized comparison of horizontal and vertical banded gastroplasty: what determines weight loss? Scand J Gastroenterol 1989;24:186-192.

12 Sjöström L, Lindross AK, Peltonen M, Torgerson J, Bouchard C, Carlsson B, Dahlgren S, Larsson B, Narbro K, Sjöström CD, Sullivan M, Wedel H: Lifestyle, diabetes and cardiovascular risk factors 10 years after bariatric surgery. N Engl J Med 2004;351:2683-93.

13 Buchwald H: Consensus conference statement. Bariatric surgery for morbid obesity: health implications for patients, health professionals, and third-party payers. Surg Obes Related Dis 2005;1:371-81.

14 Buchwald H, Avidor Y, Braunwald E, Jensen MD, Pories W, Fahrbach K, Schoelles K: Baritatric surgery. A systematic review and meta-analysis. JAMA 2004;292:1724-37.

15 Weiner S, Weiner RA, Rosenthal A, Pomhoff I: Lebensqualität nach Adipositas-Chirurgie: Ergebnisse nach verschiedenen Operationsverfahren - erste Ergebnisse einer prospektiven
Längsschnittstudie. Chir Gastroenterol 2007; 23(suppl 1):DOI 10.1159/000098160.

16 Stroh C, Manger T: Studie zur Qualitätskontrolle der operativen Therapie der Adipositas. Dtsch Ges Chir Mitteil 2004;33:389-91.

17 Stroh C, Flade-Kuthe R, Herbig B, Höhne S, Köhler H, Pick P, Horbach T, Weiner RA, Wolf S, Wolf AM, Schmidt U, Manger T, für die Arbeitsgruppe Adipositaschirurgie: Studie zur Qualitätskontrolle der operativen Therapie der Adipositas - Ergebnisse der Pilotphase 2005. Chir Gastroenterol 2007;23(suppl 1): DOI 10.1159/000098345.

18 Gärtner D, Hoyer M, Hornung A, Andus T, Bischoff S, Hesse U: Adipositaschirurgie: Ablehnung der Kostenübernahme trotz ärztlicher Gutachten. Dtsch Ges Chir Mitteil 2006;35:91-96.

19 Sauerland S, Angrisani L, Belachew M, Chevallier JM, Favrettii F, Finer N, Fingerhut A, Caballero MG, Macias JAG, Mittermair R, Morino M, Msika S, Rubino F, Tacchino R, Weiner R, Neugebauer EAM: Evidence-based guidelines of the European Association for Endoscopic Surgery (E.A.E.S.) Surg Endosc 2005;19:200-21.

20 Deutsche Gesellschaft für Chirurgie der Adipositas: Evidenzbasierte Leitlinie der chirurgischen Therapie der Adipositas 2006, pp 2-19. www.adipositas-gesellschaft.de 